\title{
NLMS Adaptive Digital Filter with a Variable Step Size for ICS (Interference Cancellation System) RF Repeater
}

\author{
Jin-Yul Kim* and Sung-Joon Park \\ Dept. of Electronic Eng. University of Suwon, Broadcom Corporation, Seoul Korea \\ jykim@suwon.ac.kr,psj1643@gmail.com
}

\begin{abstract}
In the NLMS (normalized least mean square) adaptive filters, choosing a suitable step size is crucial as the stability and the steady-state convergence error of the adaptive filter heavily depends on the step size value. In this paper, we propose a new VSS (variable step size) control method for the NLMS adaptive filter applicable to on-frequency RF repeaters that operate under fading feedback multipath channel. In the proposed method, the step size is obtained by multiplying the two step sizes. The first one adapts fast based on the estimated RFR (received-to-feedback signal power ratio) value, while the second one is adjusted slow according to the estimated fading rate of the feedback multipath channel. Computer simulations show that by adopting the proposed VSS control method the RF repeater can track the time-varying feedback channel with a good convergence error while previous VSS control methods suffer from performance degradation.
\end{abstract}

Keywords: on-frequency RF repeater, NLMS adaptive filter, variable step size

\section{Introduction}

In wireless communication system and digital broadcast systems, the on-frequency RF (Radio Frequency) repeaters are installed to clear shadowing areas existing between base stations and mobiles [1-4]. However, on-frequency RF repeaters with high RF gain usually suffer from the oscillation problem caused by the unwanted feedback signal paths from the output of the RF repeater's transmit antenna to the receive antenna due to insufficient antenna separation. The so-called ICS (interference cancellation system) RF repeater synthesizes the feedback signal by estimating the feedback signal paths using an ADF (Adaptive Digital Filter) and then subtracts the unwanted feedback signal from the receive antenna signal. The ICS RF repeater can provide more antenna separation and thus can operate stably with higher $\mathrm{RF}$ gain than the conventional RF repeater. Various adaptive filtering algorithms have been reported to cancel the interference in ICS RF repeater [5].

One of the most widely used adaptation algorithms in the ADF of the ICS repeater is the well-known LMS (Least Mean Square)-based algorithm [10] due to its simplicity and good tracking capabilities. However it is well-known that, in the LMS-based adaptive filters, choosing a suitable step size is crucial as the stability and the steady-state convergence error of the adaptive filter heavily depends on the step size value.

Many researches have been to control the variable step size (VSS) [6-9] which, however, do not consider the multi-path fading nature of the feedback channel in developing the algorithm. In this paper, we propose a new variable step size control method for the NLMS adaptive filter in the ICS RF repeaters that operate under fading feedback multipath channel.

* Corresponding Author 
The proposed method aims to improve the performance of the previous VSS NLMS adaptive filters further.

In the proposed method the step size is obtained by multiplying the two step sizes obtained based on different design considerations. First, the first step size is computed based on the estimated RFR (received-to-feedback signal ratio) value. Second, the fading condition of the feedback multipath channel is monitored using the estimated feedback signal and the second step size is controlled slowly in time according to the fading condition. The faster is the fading of the feedback channel, the larger becomes the second step size in order to track the rapid change of the feedback channel.

In order to validate the proposed method, computer simulations have been conducted in MATLAB. We show that the proposed variable step size control method can adjust the step size of the ADF filter successfully so that the on-frequency repeater can track the timevarying feedback channel conditions with a good convergence error.

\section{Interference Cancellation System}

\subsection{System model}

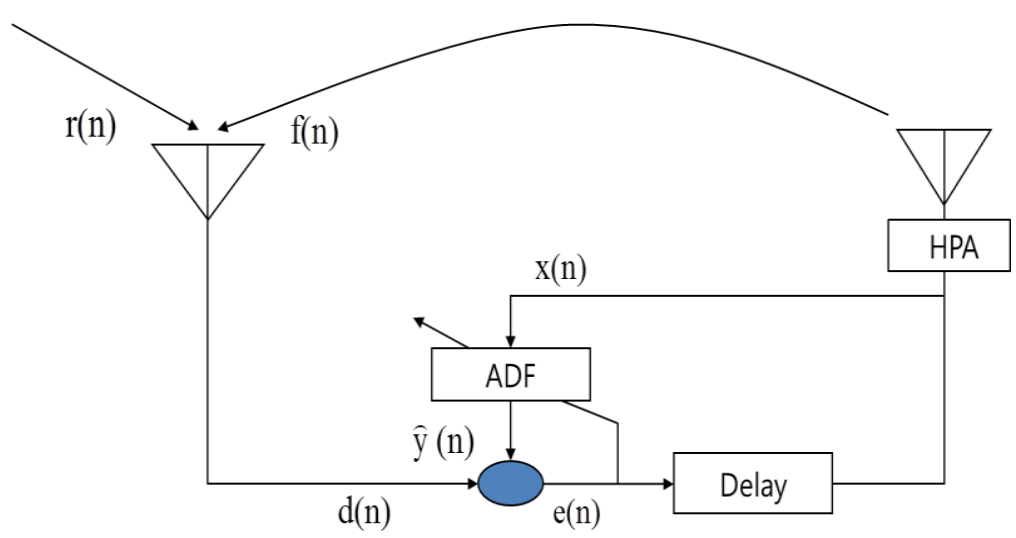

Figure 1. ICS on-frequency $R F$ repeater

Figure 1 shows the baseband model of the on-frequency RF repeater with feedback echo cancellation. The signal $r(n)$ is the received signal from the base station, $f(n)$ is the feedback interference echo signal from the transmit antenna to the receive antenna. The echo signal must be removed to assure the stability of the ICS without oscillation. $d(n)$ denotes the sum of the received signal and the interference echo signal. The ADF computes the estimate of the feedback signal, $\hat{y}(n)$, which is subtracted from the desired signal $d(n)$ to yield the error signal $e(n)=d(n)-\hat{y}(n)$. If the estimation is perfect, $e(n)$ would be consisted of the signal from the base station only. Note that since, after a bulk of delay, the e(n) can be considered uncorrelated with $\mathrm{r}(\mathrm{n})$ the filter taps of $\mathrm{ADF}$ can be trained using the filter input $\mathrm{x}(\mathrm{n})=$ $\operatorname{Delay}(\mathrm{e}(\mathrm{n}))$.

\subsection{Normalized Least Mean Square (NLMS) Algorithm}

NLMS ADF filter [10] is widely adopted due to its simple structure and tracking stability. The filter taps $\mathrm{w}(\mathrm{n})$ are updated according to:

$$
\mathrm{w}(\mathrm{n})=\mathrm{w}(\mathrm{n}-1)+\frac{\mu}{\|\mathrm{x}(\mathrm{n})\|^{2}} e(n) x(n)
$$




$$
\begin{aligned}
& \mathrm{e}(\mathrm{n})=\mathrm{d}(\mathrm{n})-\hat{y}(n), \\
& \hat{y}(n)=W^{T}(\mathrm{n}) \mathrm{X}(\mathrm{n})=\sum_{i=0}^{N-1} w(i) x(n-i) .
\end{aligned}
$$

where $\mu$ is the step size of the filter, $x(n), d(n), \hat{y}(n)$ is the filter input, the desired signal, and the filter output at time $\mathrm{n}$ respectively. It is well-known that the optimal step size depends on the power of the filter signals. Thus, instead of using a fixed step size, $\mu$ can be controlled every time step $\mathrm{n}$ [6-9]. Recently, in [9], the choice of $\mu(\mathrm{n})=\left(1-\sigma_{\mathrm{rv}} / \sigma_{\mathrm{e}}\right)$ has been proposed where $\sigma_{\mathrm{rv}}$ is the power of the sum of received signal and the system noise, and $\sigma_{\mathrm{e}}$ is the power of the error signal.

Although the adoption of the variable step size in NLMS algorithms can improve the performance of the ADF for certain circumstance, as we will show shortly through computer experiments, they do not consider the fading characteristic of the feedback channel while adjusting the step size. The echo signal $\mathrm{f}(\mathrm{n})$ usually suffer from a severe Rayleigh fading as the transmitted output signal from the transmit antenna travels through many different paths to the receive antenna and the signal components of each path are added together at the receive antenna. The previous VSS NLMS algorithms only consider the power of signals in the filter, and they do not effectively handle the fading effect in the feedback channel.

\section{The Proposed VSS NLMS ADF for on-frequency RF Repeater}

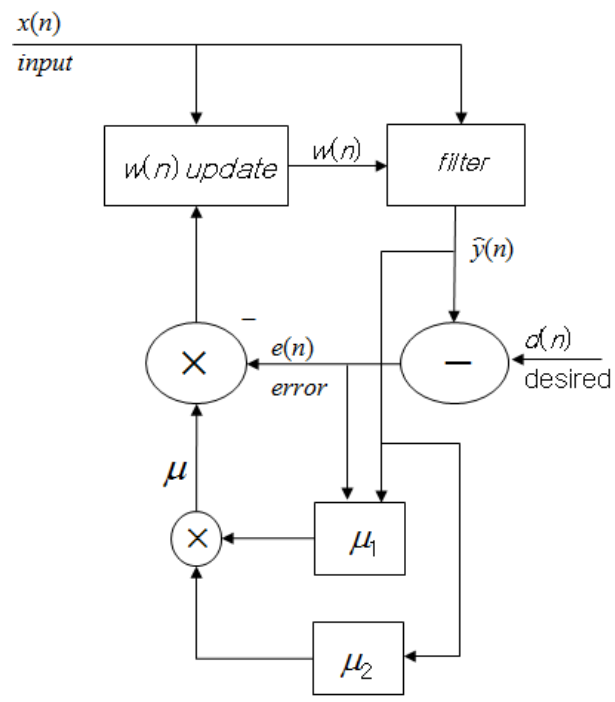

Figure 2. The block diagram of the proposed VSS NLMS filter

The block diagram of the proposed VSS NLMS filter is shown in Figure 2. The final step size $\mu$ is obtained by multiplying two step sizes, $\mu_{1}(n)$ and $\mu_{2}(n)$, obtained independently as follows. That is, $\mu(n)=\mu_{1}(n) \mu_{2}(n) \cdot \mu_{1}(n)$ is obtained as in conventional ways by considering the power of the filter signals. The VSS adjustment technique depicted in [7] has been adopted to compute $\mu_{1}(\mathrm{n})$ which is based on the ratio of the power of the estimated received signal and the power of the estimated feedback signal. Let us define the received-tofeedback signal power ratio (RFR) at time n:

$$
\operatorname{RFR}(\mathrm{n})=10 \log \frac{P_{R}}{P_{F}},
$$


where

$$
\begin{aligned}
P_{R} & =\sum_{j=0}^{M-1}[d(n-j)-\hat{y}(n-j)]^{2}=\sum_{j=0}^{M-1} e(n-j)^{2}, \\
P_{F} & =\sum_{j=0}^{M-1} \hat{y}(n-j)^{2} .
\end{aligned}
$$

Using RFR(n) the step size $\mu_{1}(n)$ is adjusted as follows:

$$
\mu_{1}(n)=\left\{\begin{array}{l}
\mu_{\max }, \text { if } R F R(n)>R F R_{\max } \\
\mu_{\min }, \text { if } R F R(n)<R F R_{\min } \\
g(n), \quad \text { otherwise }
\end{array} ;\right.
$$

where

$$
g(n)=a \cdot R F R(n)+b
$$

and

$$
\begin{aligned}
& \mathrm{a}=\frac{\mu_{\max }-\mu_{\min }}{R F R_{\max }-R F R_{\min }} \text { (gradient) } \\
& b=\mu_{\text {min }}-a \cdot R F R_{\min } \text { (bias). }
\end{aligned}
$$

Basically the step size $\mu_{1}(\mathrm{n})$ varies linearly in proportion to the measured RFR(n). To guarantee the prevention of divergence of the RF repeater we assumed the RF repeater is undergoing fast fading condition initially. The limit of the maximum and the minimum value of the step size and RFR values are selected assuming this situation.

The second step size $\mu_{2}(\mathrm{n})$ is adjusted to cope with the channel variation due to the fading. The degree of the feedback channel fading is estimated by computing the mean of the estimated variance of the feedback signal, where the filter output $\hat{y}(n)$ is used in calculation for the feedback signal.

$$
\begin{aligned}
& \widehat{V}(n)=\operatorname{var}(\text { feedback signal }) \\
& M_{V}(n)=\sum_{j=0}^{K-1} \operatorname{var}(\widehat{V}(n-j))
\end{aligned}
$$

The step size $\mu_{2}(\mathrm{n})$ is controlled to vary linearly from 0.1 to 1.0 . If the feedback channel suffers from fast fading a large step size is assigned, while if it suffer from slow fading a smaller value is assigned. The faster is the fading of the feedback channel, the larger becomes the second step size in order to track the rapid change of the feedback channel.

A procedure to adjust $\mu_{2}(\mathrm{n})$ in a simple way is shown below which has been applied to the computer simulations conducted in this paper.

Procedure: Adjust $\mu_{2}(\mathrm{n})$

1) Initial value:
A. $\mu_{2}(n)=1.0$;

2) At time step $n$;

A. Estimate the channel using $\mathrm{M}_{\mathrm{v}}(n)$;

B. If $\left(\mathrm{M}_{\mathrm{v}}(n)>T H_{-}\right.$fast $) \mu_{2}(\mathrm{n})+=\varepsilon$; else if $\left(\mathrm{M}_{\mathrm{v}}(n)<T H_{-}\right.$slow $) \mu_{2}(\mathrm{n})-=\varepsilon$;

C. If $\left(\mu_{2}(\mathrm{n})<0.1\right) \mu_{2}(\mathrm{n})=0.1$; else if $\left(\mu_{2}(\mathrm{n})>1.0\right) \mu_{2}(\mathrm{n})=1.0$; 
$\varepsilon$ is a small step value ( $=0.1$ for example). TH_fast and TH_slow are threshold values for judging fast fading condition and slow fading condition, respectively.

\section{Computer Experiments}

Figure 3 shows the block diagram of the on-frequency RF repeater for simulation. Computer simulation was done using MATLAB. The signal from the base station is randomly generated and QPSK modulated. Sampling frequency was 1usec. Major parameters used in the simulations are listed in Table 1. The NLMS filter has 200 taps and was realized by using fixed-point arithmetic for $\mathrm{H} / \mathrm{W}$ implementation consideration. In the simulations, the transmit output signal gain was set to $80 \mathrm{~dB}$ and the feedback path gain to $-60 \mathrm{~dB}$. The feedback path gain amounts to the gain due to antenna separation between transmit and receive antenna. The multipath fading model of the received signal and the feedback channel is shown in Table 2. The Doppler shift frequency of the feedback channel was set to $10 \mathrm{~Hz}$ or $100 \mathrm{~Hz}$. The variable step size input to the NLMS block is computed by multiplying the two step sizes, that is, $\mu(n)=\mu_{1}(n) \mu_{2}(n)$, where $\mu_{1}(n)$ and $\mu_{2}(n)$ are obtained as explained above.

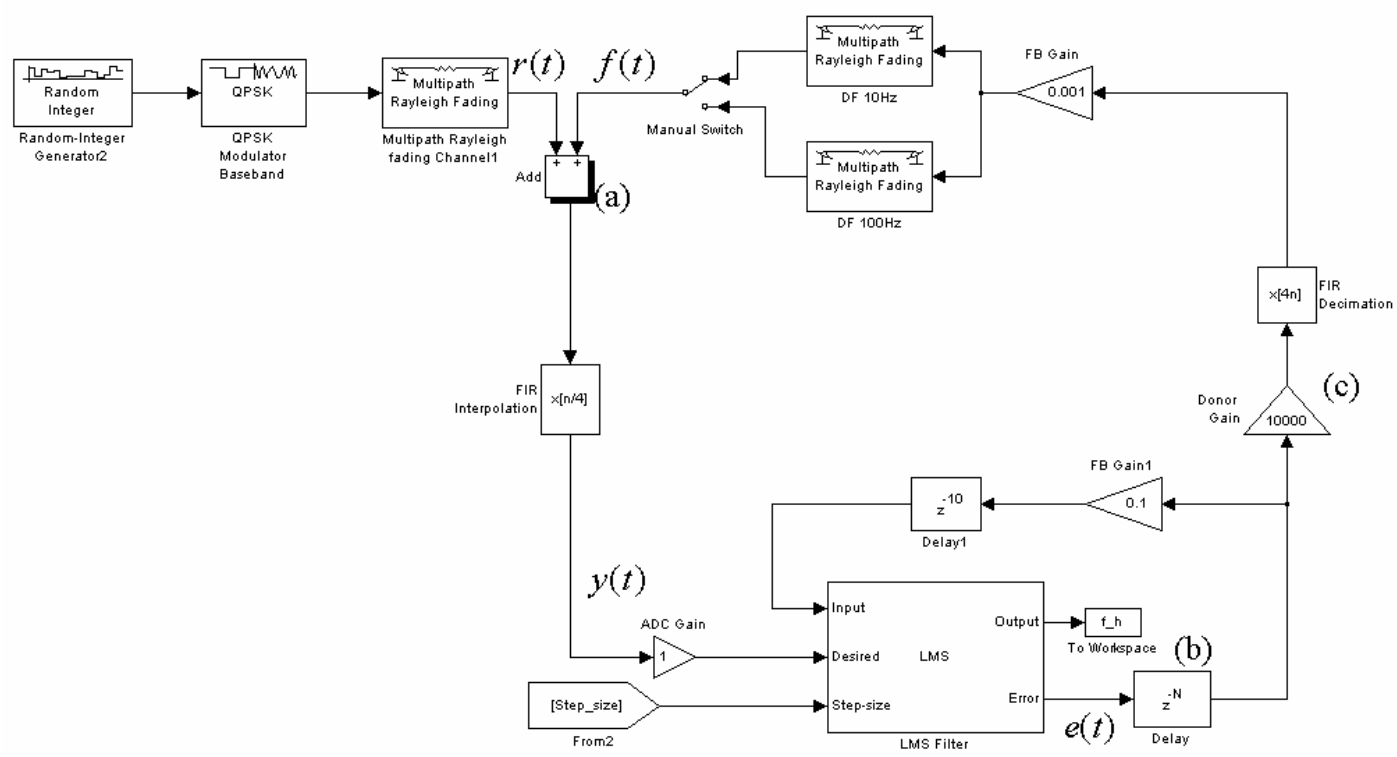

Figure 3. The block diagram of the on-frequency RF repeater for computer simulation

Table 1. The parameters for simulation

\begin{tabular}{|c|c|c|c|c|}
\hline Filter length & \multicolumn{3}{|c|}{200} \\
\hline \multicolumn{2}{|c|}{ Leakage factor } & \multicolumn{3}{|c|}{1.0} \\
\hline \multicolumn{2}{|c|}{ Initial filter weight } & \multicolumn{3}{|c|}{0} \\
\hline \multirow{2}{*}{$\begin{array}{c}\text { Fixed } \\
\text { point }\end{array}$} & Rounding mode & Nearest & Weights (bits) & $\begin{array}{c}\text { Signed Word Length : 18 } \\
\text { Fraction Length : 17 }\end{array}$ \\
\cline { 2 - 5 } & Overflow mode & Saturate & Accumulators (bits) & $\begin{array}{c}\text { Signed Word Length : 30 } \\
\text { Fraction Length : 29 }\end{array}$ \\
\hline
\end{tabular}


Table 2. Channel models for the received signal and feedback signal

\begin{tabular}{|c|c|c|}
\hline Channel Model & $\begin{array}{c}\text { Received Signal } \\
\text { (Multipath Rayleigh } \\
\text { Fading Channel) }\end{array}$ & $\begin{array}{c}\text { Feedback Signal } \\
\text { (Multipath Rayleigh } \\
\text { Fading Channel) }\end{array}$ \\
\hline Maximum Doppler shift $(\mathrm{Hz})$ & 10 & 10,100 \\
\hline Sample time & $1 / 10 \mathrm{e} 6$ & $1 / 10 \mathrm{e} 6$ \\
\hline Delay vector & {$[70 \mathrm{e}-8120 \mathrm{e}-8]$} & {$[50 \mathrm{e}-8$ 80e-8 100e-8 150e-8 250e-8] } \\
\hline Gain vector & {$[0-3]$} & {$[0-36-9-10]$} \\
\hline
\end{tabular}

\subsection{The performance of the previous VSS control method}

For comparison purpose the performance of the previous VSS control method [7] was evaluated under varying fading channel condition. For simulations the parameters were set to $R_{F R} R_{\text {min }}=-20 \mathrm{~dB}, R_{F R} R_{\text {max }}=20 \mathrm{~dB}$. The performance is evaluated by using the following measure:

$$
\operatorname{SNR}=10 \log \left(\frac{\|\mathrm{e}(\mathrm{n})\|^{2}}{\|\mathrm{r}(\mathrm{n})\|^{2}}\right) .
$$

First, with $\mu_{\min }=0.01$ and $\mu_{\max }=0.5$, the SNR is computed when the Doppler shift frequency is $10 \mathrm{~Hz}$ and $100 \mathrm{~Hz}$, respectively, and is shown in Figure 4. In the figure, the solid line denoted by 'VSS' is the result from the previous VSS control method [7] and other lines are obtained by using the fixed step sizes listed in the legend, e.g., $\mu_{1}(\mathrm{n})=0.01,0.03, \ldots$, and 0.5 , respectively. Figure 4(a), obtained for Doppler shift frequency of $10 \mathrm{~Hz}$, shows that the choice of the fixed step size 0.05 is near optimal in this situation, however we can see that the previous VSS method cannot provide solution close to this point. (Note that the fixed step size simulations are just for performance comparison purpose, and in practical system there exist no ways to obtain the optimal step size in advance.) On the contrary, Figure 4(b) shows that the previous VSS method can give near optimal performance for Doppler shift frequency of $100 \mathrm{~Hz}$.

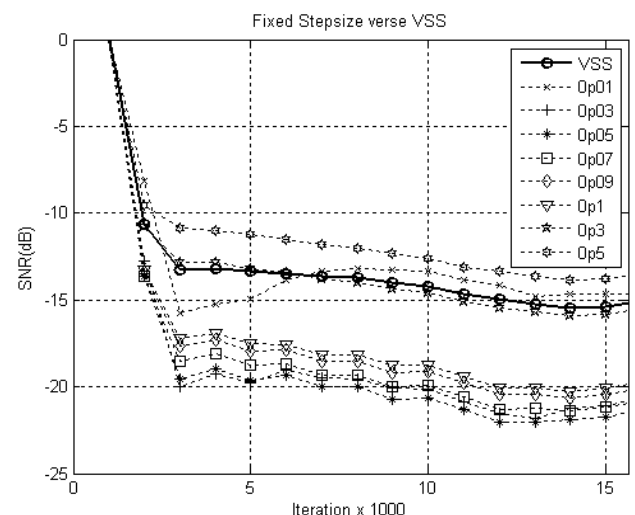

(a)

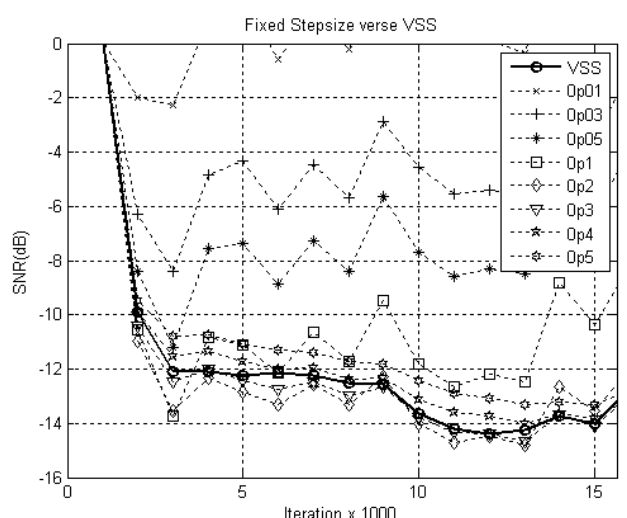

(b)

Figure 4. Previous VSS control with $\mu_{\min }=0.01, \mu_{\max }=0.5$ : (a) SNR when Dopper shift frequency is $10 \mathrm{~Hz}$, (b) SNR when Dopper shift frequency is $100 \mathrm{~Hz}$ 
In order to alleviate the performance degradation depicted in Figure 4(a), when the Dopper shift frequency is small, we can try to adjust some simulation parameters. The SNR with a choice of much smaller $\mu_{\max }=0.05$ is plotted in Figure 5(a) and (b) for the Doppler shift frequency of $10 \mathrm{~Hz}$ and $100 \mathrm{~Hz}$, respectively. In this case, however, we can observe that though the choice of a smaller $\mu_{\max }$ improve the performance for the feedback channel with a small fading rate (Figure 5(a)), the performance with a large fading rate degrades severely (Figure 5(b)).

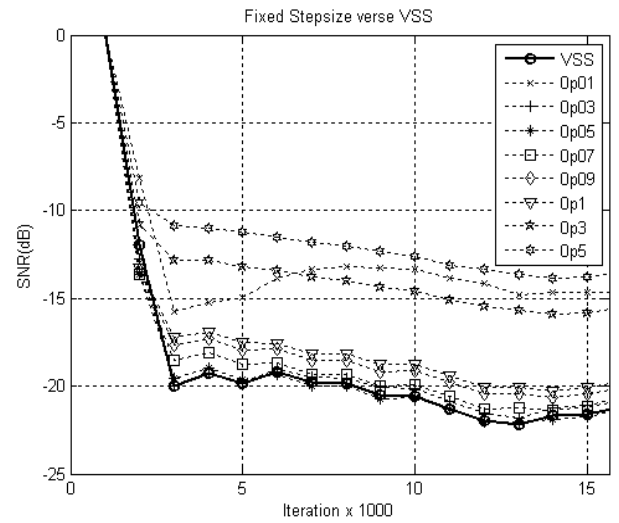

(a)

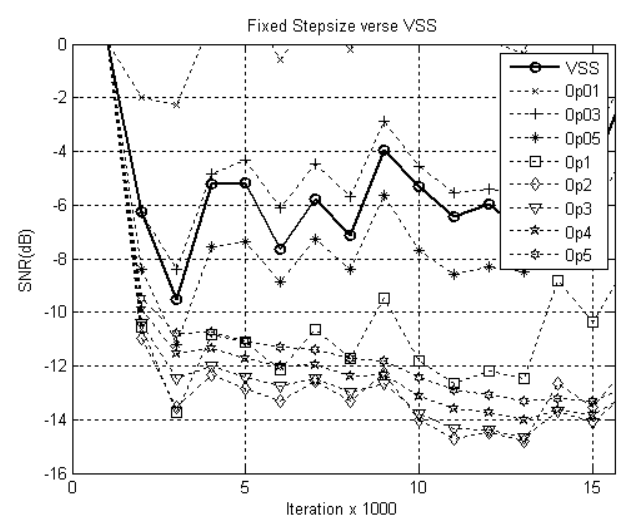

(b)

Figure 5. Previous VSS control with $\mu_{\min }=0.01, \mu_{\max }=0.05$ : (a) SNR when Dopper shift frequency is $10 \mathrm{~Hz}$, (b) SNR when Dopper shift frequency is $100 \mathrm{~Hz}$

We can see that each of the parameter setup for plotting SNR in Figure 4 and 5 is suitable for one of a fast or a slow fading feedback channel condition only, and thus the previous VSS method cannot offer satisfactory results in controlling the step size against varying fading rate of the feedback channel.

\subsection{The performance of the proposed VSS control method}

To evaluate the effectiveness of the proposed VSS control method we have conducted additional computer simulation with the same condition except the additional step size adjustment using the second step size $\mu_{2}(\mathrm{n})$.

The degree of the fading of the feedback is estimated by measuring the variance of the estimated feedback signal. An example of the measured variance of the feedback signal for Doppler shift frequency of $10 \mathrm{~Hz}$ and $100 \mathrm{~Hz}$ is shown in Figure 6. We can observe a small variance in the estimated feedback signal for a low Doppler shift frequency, while a larger one for a higher Doppler shift frequency. By averaging these estimated variances during a predefined interval the mean of the estimated variance of the feedback signal, $M_{V}(n)$ given by Eq. (12), is obtained and then the step size $\mu_{2}(\mathrm{n})$ is adjusted according to the procedure as described previously in Section 3.

Computer simulations have been conducted for the proposed VSS control method with varying fading rate of the feedback channel. The resulting SNR plot, when Dopper shift frequency is $10 \mathrm{~Hz}$, is shown in Figure 7(a), where we can see the step size gradually moves to the optimal value which in the previous VSS method was not attainable. In addition, the SNR plot when Dopper shift frequency is $100 \mathrm{~Hz}$ is shown in Figure 7(b). Also we can see the proposed VSS control method can provide near optimal step size. 


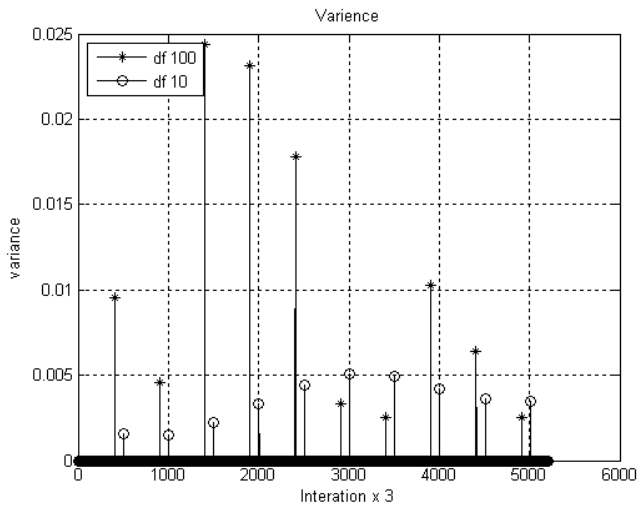

Figure 6. Measured variance of the feedback signal for Doppler shift frequency $10 \mathrm{~Hz}$ and $100 \mathrm{~Hz}$

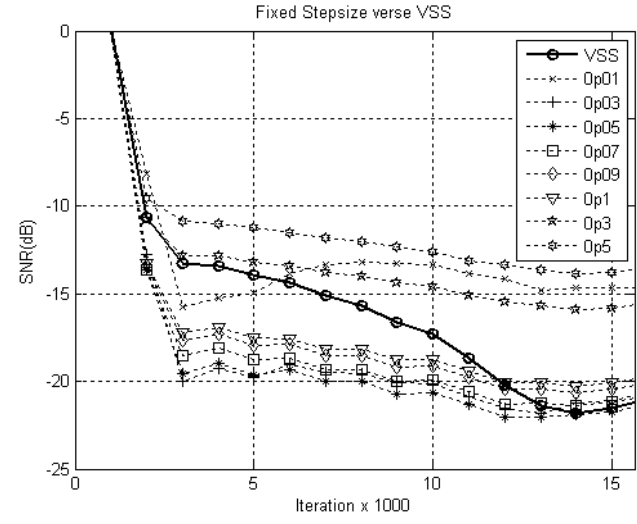

(a)

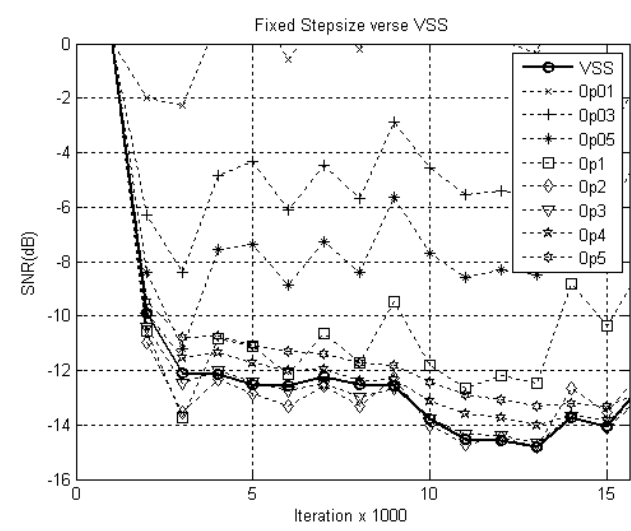

(b)

Figure 7. Proposed VSS control with $\mu_{\min }=0.01, \mu_{\max }=0.5$ : (a) SNR when Dopper shift frequency is $10 \mathrm{~Hz}$, (b) SNR when Dopper shift frequency is $100 \mathrm{~Hz}$

Finally, computer simulations have been conducted to compare the tracking capabilities of the VSS controlling methods under abrupt change of the channel condition (Figure 8). In this experiment we shifted the fading rate of the feedback channel abruptly from $10 \mathrm{~Hz}$ to $100 \mathrm{~Hz}$ near 22-th iteration step, and back to $10 \mathrm{~Hz}$ at 43 -th iteration step.

Thick solid line is the result from the proposed VSS method, while the lines with a triangle and a circle are from the fixed step size method ( 0.1 and 0.03 , respectively), and the line with a small black dot is obtained from the previous VSS method. We can see that the previous VSS method performs well at fast fading condition only and ADF with the fixed step size performs well at slow fading condition only. However, the proposed method shows good performance for both the fading condition in spite of abrupt channel variation. This graph confirms that our proposed method is working well in various channel status. 


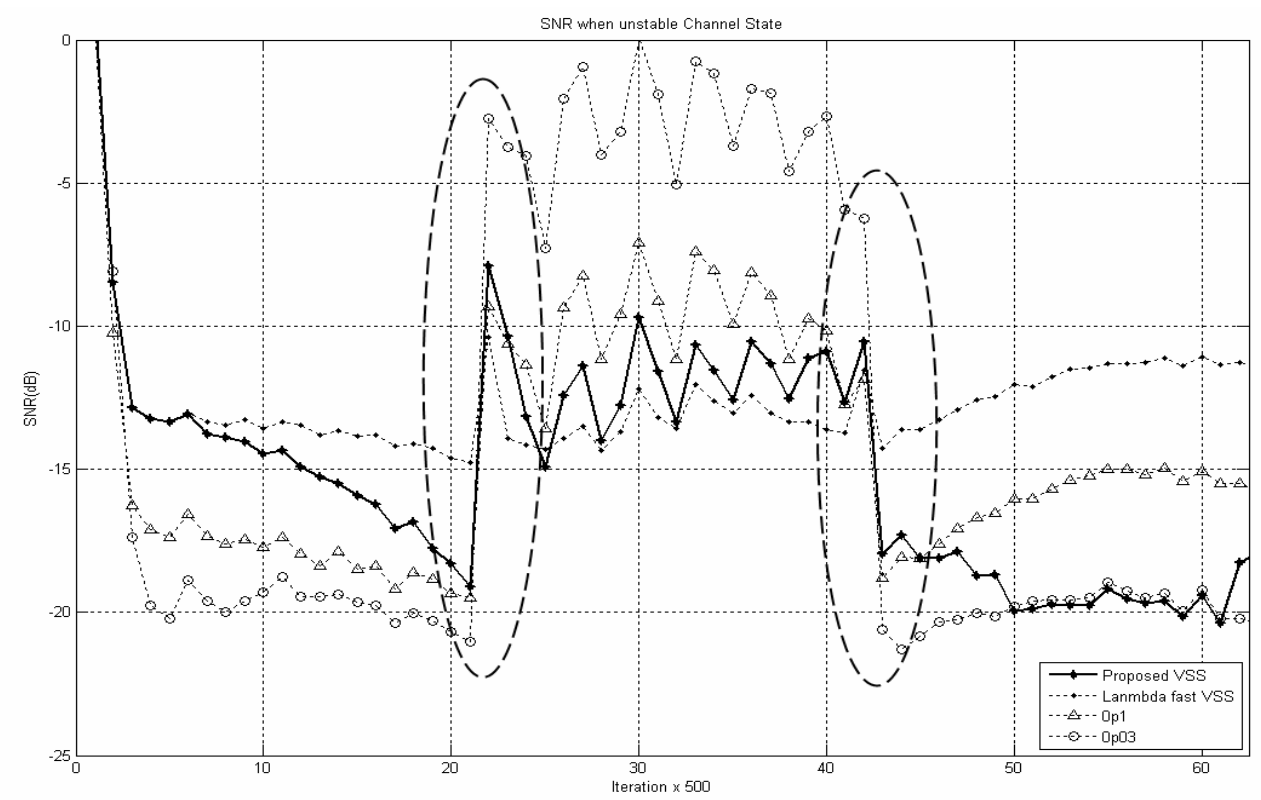

Figure 8. Comparison of the performance under changing feedback channel fading rate: The fading rate has been abruptly shifted from $10 \mathrm{~Hz}$ to $100 \mathrm{~Hz}$ near 22 iteration step, and shifted back to $10 \mathrm{~Hz}$ at 43 iteration step

\section{Conclusions}

We proposed a new variable step size control method for NLMS adaptive filter in the onfrequency RF repeaters that operate under fading feedback multipath channel. In the proposed method the step size is obtained by multiplying the two step sizes. The first step size is for fast adaptation and is computed based on the estimated RFR. The second step size reflects the fading condition of the feedback multipath channel and is controlled slowly in time according to the fading condition. Computer simulations show that by adopting the proposed VSS control method the RF repeater can track the time-varying feedback channel conditions with a good convergence error while the previous VSS control methods suffer from severe performance degradation.

\section{Acknowledgements}

This work was partly supported by the GRRC program of Gyeonggi province [GRRC Suwon 2013-B2, Center for U-city Security \& Surveillance Technology].

\section{References}

[1] H. Odate, "A frequency offset booster with an oscillatory prevention function for land mobile communication", IEEE VTC, (1987), pp. 430-434.

[2] H. Sato, K. Itoh, Y. Ebine and M. Sato, "A booster configuration with adaptive reduction of transmitterreceiver antenna coupling for pager systems", IEEE VTC, (1999) September, pp. 1516-1520.

[3] K. Shibuya, "Broadcast-Wave Relay Technology for Digital Terrestrial Television Broadcasting", Proceeding of the IEEE, vol. 94, no. 1, (2006), pp. 269-273.

[4] H. Hamazumi, K. Imamura, N. Iai, K. Shibuya and M. Sasaki, "A study of a loop interference canceller for the relay stations in an SFN for digital terrestrial broadcasting”, IEEE Globecom, vol. 1, (2000) November, pp. 167-171. 
[5] J. Dai, Z. Han and F. Zhang, "Research on the Interference Cancellation Based on Adaptive Algorithms", Journal of Intelligent Engineering and Systems, vol. 5, no. 4, (2012).

[6] H. C. Woo, "Variable Step Size LMS Algorithm Based on Error Criteria", Journal of Information \& communication, vol. 5, no. 1, (2007).

[7] J. -K. Lee, J. -H. Park and C. -W. Lee, "Research about Adjusted Step Size NLMS Algorithm Using SNR", The Journal of Korea Information and Communications Society (J-KICS), vol. 33, no. 4, (in Korean), (2008).

[8] C. Liu, W. Xia, Z. He, R. Qian and J. Zhou, "Adaptive Feedback Interference Cancellation Algorithm for Digital On-channel Repeaters in DTTB Networks", 3rd International Conference on Computer and Electrical Engineering (ICCEE 2010), (2010).

[9] Z. Liua, W. Xia, Z. He, C. Liu and J. Zhou, "A VSS-NLMS Algorithm Designed for DTMB On-Channel Repeater", International Conference on Computer Science and Information Technology (ICCSIT 2011), (2011).

[10] S. Haykin, “Adaptive Filter Theory”, 4th ed., Prentice-Hall, Inc., (2002).

\section{Authors}

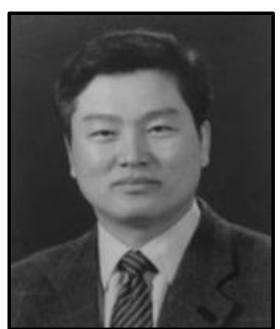

Jin-Yul Kim received a BA degree in Electronics Eng. from Seoul National University in 1986, and MA degree and Ph.D degree in Electrical Eng. from Korea Advanced Institute of Science and Technology, in 1988 and 1993, respectively. He is an associate professor at Dept. of Electronic Eng., University of Suwon, South Korea. His research interests include signal processing for digital communications, image processing, and visual object tracking.

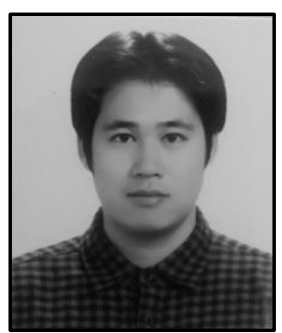

Sung-Joon Park received BA degree and MA degree in Electronics Eng. from University of Suwon, South Korea, in 2007 and 2009, respectively. He is currently working for Broadcom Corporation, South Korea, and his areas of interest include wireless communications, software and hardware design for wireless modem. 